\title{
31
}

\section{Flexible assignment submission in distance learning}

\author{
Rod Byrnes \\ Bruce Lo \\ Julian Dimbleby \\ Southern Cross University \\ New South Wales \\ Australia
}

\begin{abstract}
Distance learning students and internal students enrolled in any course at Southern Cross University may now take advantage of the flexibility provided by the Assignment Management System. Using this system, students may receive assignment specifications, submit completed assignments in many formats (text, graphics, multimedia) and receive marked, annotated assignments from their home personal computer or from any networked computer on campus. This easy-to-use system significantly reduces time delays associated with the conventional assignment-return cycle and eliminates the need for paper documents. Instructors avoid the problems associated with managing assignments and record keeping, since these tasks are now automated. The flexibility of this client-server system encompasses many areas: more time for assignment submission, no restriction on the student's geographical location, flexibility in assignment size and format, marks and grades automatically handled, and flexibility for future system growth to add new features.
\end{abstract}

Main conference themes: flexible learning

Educational areas: higher education

Study topics:

Secondary keywords: electronic mail, home learning, management, open learning, telecommunications 


\section{INTRODUCTION}

In those institutions where distance learning programs have been introduced, information technology (IT) has facilitated the learning activities of students in a variety of ways. These IT applications can be categorized into those which deliver instructional content such as Computer Aided Learning (CAL); those which provide a mechanism for assessment, such as Computer Based Testing (CBT); those which support administrative activities in learning, such as Computer Managed Learning (CML); and those which provide enhanced communication facilities between students and instructors, namely Computer Mediated Communications (CMC). Although this classification is not unique and the different categories overlap, it still serves as a useful means to describe the application of information technology in learning.

In the majority of distance learning programs, the forms of assessment handled by the technology are limited (text based, short answer, multiple choice questions, etc.) and are often not appropriate for the different disciplines involved. As such, the conventional written assignment has remained a predominant method of assessing the competency of distance students.

The major difficulty with this type of assessment is the large feedback delays incurred by using the postal system for the two-way delivery of assessment material between students and their instructors. Attempts have been made to overcome this with the aid of computers, but the solutions have often been plagued by such things as:

- difficult to use;

- limited to text based material (not allowing graphics or other format);

- poor annotation facilities.

This paper describes a flexible assignment submission and management system which was developed in order to address these problems, and will concentrate on the advantages which this system can offer to students studying at a distance.

The main part of this project may be viewed as an attempt to provide a more flexible learning environment for learners. Before giving a more detailed description of the system itself, we shall first explore the concept of flexible learning.

\section{Flexible learning}

There are two questions in connection with this concept. Firstly, what is made flexible in the learning process? Secondly, for whose benefit is flexibility designed? 
The teaching-learning process involves a series of interrelated activities which may be represented as a chain of six events:

- prepare curriculum content for instruction (select, prioritize, organise, structure);

- design instructional strategy (sequence, enhance, illustrate);

- deliver instructional content (state, explain, summarise);

- reinforce learning (practice, review, experiment, apply, use);

- assess learners' knowledge (test, examine, evaluate: formative and summative);

- document learners' achievement (record, rank).

Obviously this is an oversimplification of a complex process. However, it is adequate for the purpose of the present analysis. These activities are illustrated in Fig. 1 as stages in a teaching-learning chain:

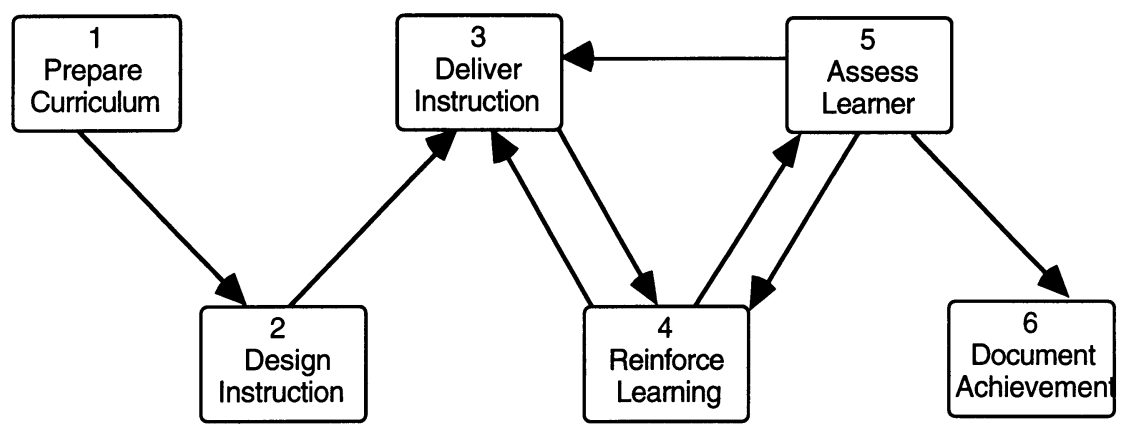

Fig. 1 The teaching-learning chain

Flexibility may be introduced at any stage of this chain. What is being made flexible depends on which stage is being targeted. Ross argued that flexibility in learning may refer to one or more of the following [1]:

- time (when and how long to learn);

- place (of study);

- qualifications (for entry to study);

- methods (of instruction);

- resources (for learning);

- problems/questions (addressed by students in their learning);

- content (selected by learners);

- learning methods. 
The present study concentrates on the role of assignments in the teachinglearning process-stages 3,4 and 5 in Fig. 1. Therefore, flexibility in this context can refer to:

- flexibility in time of assignment submission;

- flexibility in location for submission and return;

- flexibility in the format of assignments;

- flexibility in the amount, quality and type of feedback;

- flexibility in management of assignments;

- flexibility in the degree of interactiveness in assignments.

A flexible assignment submission system must address each of these issues and allow assignment documents to flow directly from students to instructors, and vice versa, without time or geographical constraints. To optimize the value of assignments in the teaching-learning chain, the format of the assignment must be appropriate to the discipline being taught. For example in a graphic art discipline, students must be able to include graphics files in their assignment.

The activities to be undertaken in stages 3,4 and 5 of the teaching-learning chain have been expanded in Fig. 2. This figure distinguishes those assignment related activities which are undertaken by instructors and those by students. Also indicated in the figure are those activities which are supported by the assignment submission system under consideration.

\section{Review of Computer Managed Learning (CML)}

The flexible assignment submission system developed may be considered as a particular example of Computer Managed Learning (CML), which has been defined as 'the application ... where the function of the computer is in the control, administration and testing of the learning process' [2,3]. McCrae [4] notes four areas in which CML systems currently provide support:

- constructing, marking and analyzing tests;

- keeping records of students' performance;

- providing individualized guidance;

- providing performance feedback to instructors.

An extended CML system could act as the interface or coordinator for other components in the system, and could enable instructors to spend more time teaching and developing teaching materials, and less time on administrative tasks. 
It is recognized that different authors have different views on what should compose a CML system, but the following components are a reasonable representation:

- Computerized testing and marking;

- Administrative tasks e.g. record keeping, student management and course management;

- Assignment management;

- Instructor/student communication;

- Facilities for managing and accessing CAL materials.

\begin{tabular}{|l|c|c|c|}
\hline \multicolumn{1}{|c|}{ Activity } & Instructor & Student & $\begin{array}{c}\text { Supported by } \\
\text { AMS }\end{array}$ \\
\hline Produce assignment specification & $\checkmark$ & & \\
\hline Produce marking scheme & $\checkmark$ & & \\
\hline Distribute assignment and mark scheme to students & $\checkmark$ & & $\checkmark$ \\
\hline Create template file to capture student marks & $\checkmark$ & & $\checkmark$ \\
\hline Produce assignment, using a variety of software & & $\checkmark$ & \\
\hline Submit assignment (multiple files) & & $\checkmark$ & $\checkmark$ \\
\hline Submit an amended assignment & & $\checkmark$ & $\checkmark$ \\
\hline Mark assignments, provide feedback comments & $\checkmark$ & & $\checkmark$ \\
\hline Apply penalty to "late" assignments & $\checkmark$ & & $\checkmark$ \\
\hline Enter marks into master file & $\checkmark$ & & $\checkmark$ \\
\hline Provide sample solution to all students & $\checkmark$ & & $\checkmark$ \\
\hline Broadcast comments on assignment performance & $\checkmark$ & & $\checkmark$ \\
\hline Read Instructor feedback comments & & $\checkmark$ & $\checkmark$ \\
\hline Query own mark & & $\checkmark$ & $\checkmark$ \\
\hline Query statistical profile of group & & $\checkmark$ & $\checkmark$ \\
\hline Submit an appeal for remark & & $\checkmark$ & $\checkmark$ \\
\hline Remark & $\checkmark$ & & $\checkmark$ \\
\hline Submit final grades to Administration & & $\checkmark$ \\
\hline
\end{tabular}

Fig. 2 Assignment activities: instructor's and student's role

A number of people have implemented CML systems which manage the assignment process. These systems range from 'dumb' systems merely facilitating delivery of assignments, through to 'intelligent' systems which attempt to automatically mark the assignment and give meaningful feedback to the student. Hasan [5] describes a CML system supporting assignment submission. Students submit assignments by logging on to computers attached to a LAN. In some cases, a script can be invoked automatically which 
processes the assignment and gives immediate feedback to the student, but many assignments cannot be automatically marked.

Feedback to the student in the form of annotations is an integral and extremely important aspect of paper based assignments $[6,7]$. Most electronic systems do not support this. There are essentially two different aspects to feedback: response time and content. Lyall [8] breaks this down further into five different categories: immediacy, regularity, explanatory, conciseness, and clarity.

Our system aims to provide students with a user-friendly approach to submitting assignment and obtaining feedback comments and results. This systems is described in more detail in the next section.

\section{The Assignment Management System}

Our project to develop an Assignment Management System (AMS) was initiated at Southern Cross University, Australia in 1994. This system adopted the written assignment as the basic unit of assessment and enabled students to submit assignments in electronic form into a database, and to retrieve the marked assignments along with feedback after they had been reviewed. The instructors used the same system to mark the assignments and keep track of student grades. Fig. 2 shows in more detail which activities are supported by the AMS.

The major difference between this initiative and others in the field is that the AMS provides a user friendly graphical user interface for students and instructors. Because it is based on the client/server paradigm, a high quality GUI can be used even with an ordinary modem as the client does not require large quantities of information. Communication can be done through a network or a phone line and is all handled transparently by the system.

\section{Flexibility in feedback}

Immediate feedback is of paramount importance in the learning process. Using computers and electronic communication technology to submit assignment documents can reduce the delay to literally minutes. However, problems with this approach are:

- the instructor must have the appropriate software to view the student's work;

- feedback comments from an instructor can easily be 'scribbled' on a written document, but it is not so easy when that document is stored electronically.

Two potential solutions were considered. Either a specialized software package needed to be acquired or the existing application might already contain 
annotation functionality. Adobe's Acrobat is an example of the first approach, and Microsoft Word is an example of the second approach.

\section{Flexibility at a distance}

The AMS offers a number of advantages to students studying at a distance. Submission times are more flexible. Because the students are not reliant on the postal system, they are able to submit their assignment at any time. Furthermore, if there is a deadline, the student has an extra few days in which to work on the assignment before they must submit it. Location is flexible. Students do not need to leave their house in order to submit the assignment. For students who live in remote areas such as the Australian outback, it can take hours driving over often bad roads to get to the nearest post office. With a telephone line (or satellite telephone) they are as 'close' to the university as any other student.

\section{Flexibility of Assignment Format}

Assignment format is flexible. It is not just limited to text, so the student can include charts, tables and pictures as desired. Because the assignment will be viewed on a monitor rather than be printed, colour can be utilized very effectively and inexpensively.

Another advantage is that solutions to assignments can be developed interactively by iterating between the student and instructor. This means a first draft of the solution may be proposed by the student and sent to the instructor. The instructor may comment on the first draft. The student then revises the solution as a second draft which may again be submitted. The iteration process may continue until both parties are satisfied.

\section{Flexibility for instructors}

For instructors assignment material can be uploaded onto the AMS for all students, both on and off campus. Students' work can be uploaded in the same way. This makes it easier for the instructors who have only one set of work to produce. Both on-campus students and distance students will have equal access to the assignment material.

\section{DESIGN OF THE AMS}

When designing the AMS, the key objectives were:

User friendliness This was of ultimate importance, because the AMS was intended to provide services for all students enrolled at the university. The 
interface had to be simple enough to be mastered in a few minutes by a person with only minimal computer experience.

Expandability The system should be capable of readily incorporating new features. It is anticipated that the AMS will be expanded in the future to provide more services such as automatic marking of assignments and computer based testing.

Accessibility There were four types of location from which the system should be accessible:

- On campus access from computers attached to the university network;

- Open Learning Access Centres away from the main campus;

- Off campus access through the Internet;

- Off campus access through dial up telephone lines.

Flexibility The system should provide a framework which would support a wide variety of assignment types, including text, computer programs, and documents containing diagrams, pictures, audio and video.

Figure 3 illustrates the functional structure of the AMS.

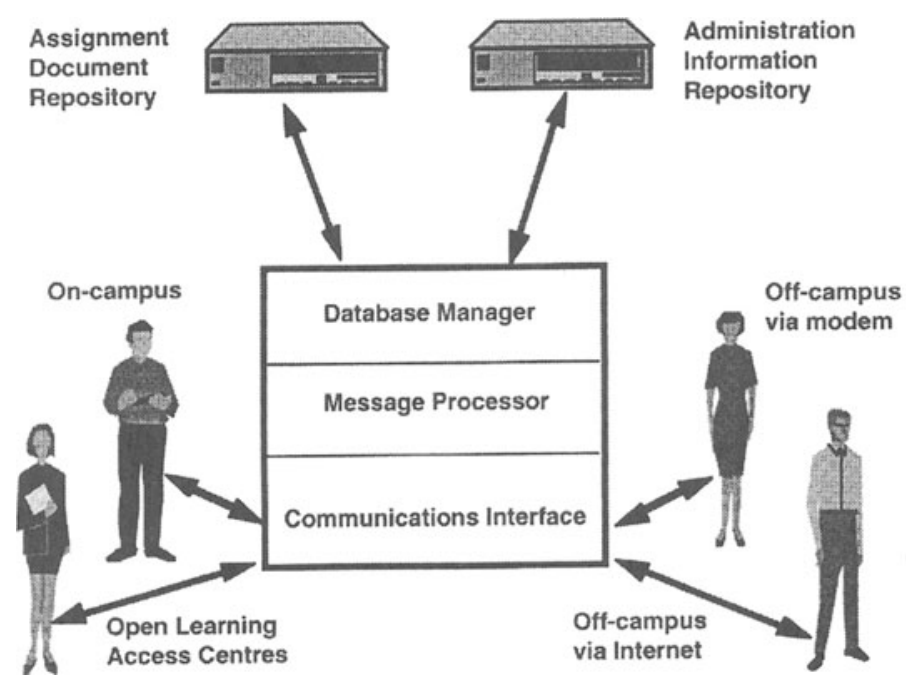

Fig. 3 Structure of the Assignment Management System 
The AMS was designed around the client-server paradigm which has a number of advantages:

- The AMS can operate on a variety of platforms, from VT100 to XWindows, by providing appropriate versions of the client software;

- User friendly GUI's can be used over slow dial-up lines, as the client computer is responsible for controlling the display. In general interfaces over dial-up lines have required the use of unattractive text based displays;

- The database can be altered at any time, and providing that the communication interface remains the same, the client program needs no modification at all;

- The client decides how to process the information. Different material like sound and graphics can be manipulated at the client end, but is still treated as raw data by the server. The server facilitates the passing of information between the clients;

- Given the right communication technologies AMS can operate over a variety of networks or dial-up lines. This is because the client and server both operate on top of existing network protocols. To migrate to a different networking technology does not require the rewriting of either the client or the server, but only the replacement of the underlying network drivers.

\section{Implementation of the AMS}

TCP/IP was chosen as the networking protocol because it is so widespread and standard. It can also be used over dial-up telephone lines.

Windows was chosen as the platform on which the initial client program was to be developed. This was because most of the laboratories at the university contain Windows based machines, Windows programming tools were available, and the project programmer was familiar with Windows. The AMS was designed to interface with third party products for the actual document management, such as creation and editing. Windows supports this use of third party products easily through its concept of filename extension associations. If a particular extension (for instance, .doc) has a program associated with it, executing the file will cause the program to be run and the file loaded immediately.

The server was built on an IBM-PC running SCO Unix. SCO Unix was chosen because it is a very stable operating system. Also it is a multitasking operating system, this being was a requirement for this application. The university already owned a very good relational database for this operating system.

There were two components to the server. The first consisted of the communications interface. This provided the logic to interface with the client 
across the network, and translate client requests into relational database commands. The second component of the server was the relational database engine itself. A database engine was used because of the flexibility and security it provided. Security is a very important aspect in a project such as this one.

When comparing this implementation to the list of requirements of the system as outlined in the previous section, we see that the system fulfils every requirement:

- User friendliness is achieved with the use of a GUI such as Windows.

- The system is expandable. The client program has been designed in a modular way with each function of the system residing in a different module. To add more functions, they only have to be linked into the existing framework. The server would only need to have the extra messages built into its communication interface, and the relevant database access code written. The database could also be expanded with minimal changes.

- The AMS is extremely accessible. Currently, it can be run from any IBMPC which has a direct Internet connection. To add other computer types, such as Macintosh, would only require the client software to be modified. The server does not need to know what type of computer is accessing it.

- Flexibility exists with document type. The AMS does not process the content of documents in any way. This is all handled at the end-user's machine. Any document format or feedback mechanism which can be handled by the end-user's machine will automatically be supported by the AMS.

Evaluation of this newly written AMS will commence in September 1994. This initial field test of the system will be undertaken with six classes of on-campus undergraduate students, and their instructors, in different subject areas and in different years of study.

\section{SUMMARY}

Distance learning students enrolled in any course at Southern Cross University may now take advantage of the flexibility provided by the Assignment Management System.

Using intuitive GUI client software on their personal computer at home or at a nearby Open Learning Access Centre, students may now receive assignment specifications, submit completed assignments in many formats and receive marked, annotated assignments. This easy-to-use system significantly reduces time delays associated with the conventional assignment-return cycle and 
eliminates the need for paper documents. Instructors now avoid the problems associated with managing assignments and record keeping, and can focus on providing quicker and higher quality feedback for students.

The flexibility of this system encompasses many areas. Flexibility in assignment submission, flexibility in geographical location, flexibility in assignment size, format and version, and ability to manage marks and grades. Also modular design of the system provides flexibility for future growth to add new features such as automated assignment marking.

\section{REFERENCES}

1. Ross, R. (1991) Structure and Functions: Implications of Access, Open Learning and Technology, in Occasional Papers in Open Learning, (ed Ellis, A.) 1, pp. 18-25.

2. Stanford, J.D., and Cook, H.P. (1987) Computer Managed Learning-Its application to increase student achievement using formative self-assessment, in Proceedings of CALITE 87, pp. 367-378.

3. Hagan, R., and Trathen, C. (1993) Multimedia E-mail for Computer Mediated Education Materials, in Proceedings of ASCILITE 93, pp. 316-323.

4. McCrae, M. (1987) The computer based education system: A process of integration? in Proceedings of CALITE 87, pp. 322-330.

5. Hasan, H. M. (1991) The Paperless Classroom, in Proceedings of ASCILITE 91, pp. 267-276.

6. Kaye, A. (1989) Computer-Mediated Communication and Distance Education, in Mindweave (eds Mason, R. and Kaye, A.), Pergamon Press, Oxford, pp. 3-21.

7. Scriven, B. (1991) Perceptions of quality in distance education, in Proceedings of the ASPESA Biennial Forum, N.S.W: Charles Sturt University, Bathurst, pp. 439-449.

8. Lyall, R. J. (1991) But is the pen mightier than the electron? Using interactive technologies in teaching chemistry at a distance, in Proc. of the ASPESA Biennial Forum, N.S.W: Charles Sturt University, Bathurst, pp. 307311. 